Revue d'histoire de l'Amérique française

Q REVUE D.HISTOIRE DE L'AMÉRIQUE FRANÇAISE

\title{
La perspective nationaliste d'Henri Bourassa, 1896-1914
}

\section{Joseph Levitt}

Volume 22, numéro 4, mars 1969

URI : https://id.erudit.org/iderudit/302828ar

DOI : https://doi.org/10.7202/302828ar

Aller au sommaire du numéro

Éditeur(s)

Institut d'histoire de l'Amérique française

ISSN

0035-2357 (imprimé)

1492-1383 (numérique)

Découvrir la revue

Citer cet article

Levitt, J. (1969). La perspective nationaliste d'Henri Bourassa, 1896-1914. Revue

d'histoire de l'Amérique française, 22(4), 567-582.

https://doi.org/10.7202/302828ar d'utilisation que vous pouvez consulter en ligne.

https://apropos.erudit.org/fr/usagers/politique-dutilisation/ 
Joseph Levitt

\section{LA PERSPECTIVE}

NATIONALISTE

\section{D'HENRI BOURASSA}

1896-1914

Richard Jones

Commentaire 


\section{Joseph Levitt}

Ph.D. de l'Université de Toronto, 1966.

Assistant-professeur à l'Université d'Ottawa.

\section{A paraître :}

The Social Program of the Nationalists of Quebec 1900-1914, thèse de Ph.D. qui sera publiée aux Presses de l'Université d'Ottawa sous le titre: Henri Bourassa and the Golden Calf. taires.

Une sélection des écrits de Bourassa accompagnée de commen-

\section{Richard Jones}

B.A. Honours de l'Université Princeton (N.J.). Maîtrise en histoire de l'Université Laval (Québec).

A enseigné deux ans au Collège de Sainte-Anne-de-la-Pocatière. Etudes doctorales présentement en cours à l'Institut d'histoire de l'Université Laval (Québec).

Auteur de :

Community in crisis - analyse du nationalisme canadienfrançais (Toronto, Macmillan, 1966). 


\section{LA PERSPECTIVE NATIONALISTE D'HENRI BOURASSA (1896-1914)}

Un amour du Canada français et un attachement pour l'Église catholique, telles étaient les forces émotives dynamiques qui dominaient chez Bourassa. Son sentiment intense de patriotisme, croyait-il, découlait non seulement des liens de l'histoire, de la religion, de la langue et de la géographie, mais encore de la "voix du sang",1 propriété mystique qu'il attribuait à sa "race". Au début du siècle présent, le mot "race" signifiait plus que les caractéristiques physiques communément héréditaires. On croyait que chacune des grandes nations de l'Europe possédait une origine commune dans le sang - qu'on était donc latin, anglo-saxon, teuton ou slave. De chaque race jaillissait une combinaison particulière de traits affectifs et mentaux qui déterminaient le caractère national de chaque peuple ou "race". Pour Bourassa, le mot "race" comprenait implicitement cette dernière attribution. Il parlait continuellement des tendances et des instincts de la race. ${ }^{2}$ Les caractéristiques de la race française, disait-il, sont l'honneur, la probité, et la persévérance. ${ }^{3}$

Le racisme de Bourassa rejoignait parfaitement sa perspective religieuse. Il croyait que Dieu destinait à des peuples entiers des missions particulières à remplir. A chacun, on avait accordé le sublime, mais périlleux don d'une volonté nationale qui leur permettait "de correspondre, plus ou moins fidèlement, à leur vocation". Chaque peuple a reçu les aptitudes physiques, mora-

1 Henri Bourassa, "Le patriotisme canadien-français", Revue canadienne, XVI $\left(\mathrm{n}^{\circ} 1\right)$ (juin 1902): 431.

2 Henri Bourassa, "Le rôle des Canadiens français", L'Action nationale, XLIII (janvier 1954) : 125, 135.

3 Henri Bourassa, "Le patriotisme canadien-français", Revwe canadienne, XVI ( $\left.\mathrm{n}^{\circ} 1\right): 437$. 
les et intellectuelles nécessaires pour remplir son rôle, ${ }^{4}$ assuraitil, et le succès de chacun dans l'exécution de la volonté de Dieu dépend de l'usage qu'il fait de ses instincts naturels.

"L'instinct de race, écrivait encore Bourassa, est un puissant moyen d'action individuelle et sociale; mais comme les autres instincts, il doit être contrôlé et tempéré par la raison." 5 Au-delà de l'épurement par la raison, l'instinct exige pour atteindre le plein essor de sa puissance d'action, des connaissances rendues nécessaires par les circonstances sociales environnantes. ${ }^{6}$ Seuls la culture ou le comportement acquis peuvent couronner le succès social. La langue est un exemple très significatif de la culture; le langage, une aptitude acquise seulement par l'instruction, mais sans cette compétence, les instincts de la race seraient démentis et cette nationalité disparaîtrait en tant qu'entité distincte. "La conservation de la langue, insistait Bourassa, est absolument nécessaire à la conservation de la race, de son génie, de son caractère et de son tempérament." 7

Bourassa croyait aussi que les Canadiens français, quoique formant une nationalité séparée ${ }^{8}$, possédaient plusieurs des mêmes aptitudes que les Français. Au plan moral, les Français avaient été choisis pour devenir "le foyer principal de la pensée chrétienne", et au plan intellectuel, pour être "la continuation du génie grec". ${ }^{9}$ Ces éléments héréditaires mettaient les Canadiens français en état d'entretenir une supériorité intellectuelle à l'égard des Anglo-Saxons, bien que ceux-ci possédassent instinctivement le génie pour les affaires. ${ }^{10}$

${ }^{4}$ Henri Bourassa, "Le rôle des Canadiens français", L'Action nationale, XLIII (janvier 1954) : 114.

5 Henri Bourassa, "Le patriotisme canadien-français", Revue canadienne, XVI $\left(\mathrm{n}^{\circ} 1\right): 434$.

6 Henri Bourassa, "Le rôle des Canadiens français", L'Action nationale, XLIII (janvier 1954) : 134.

7 Henri Bourassa, La langue française et l'avenir de notre race (Québec, 1913), 4 .

8 Henri Bourassa, "Le patriotisme canadien-français", Revue canadienne, XVI $\left(\mathrm{n}^{\circ} 1\right): 432$.

9 Henri Bourassa, La langue française et l'avenir de notre race (Québec, 1913), 4 .

10 Henri Bourassa, "Le rôle des Canadiens français", L'Action nationale, XLIII (janvier 1954) : 127. 
Comme preuve de la supériorité culturelle innée des Latins, Bourassa n'avait qu'à se tourner vers la France. L'éclat de l'art français et de sa littérature brillait aux yeux de tous; Paris était incontestablement la capitale artistique du monde. $\AA$ l'exposition de Paris de 1900, les Français étalent leurs progrès impressionnants dans les domaines de la science et de l'industrie. La France, remarque Bourassa, exalté, est "le centre intellectuel du monde".11 Ces triomphes des artistes et des hommes de science français fournissaient des raisons de plus pour que Bourassa ressente une confiance illimitée dans l'avenir de cette même culture en Amérique du Nord.

Bourassa se glorifiait alors d'être français, comme d'autres se disaient fiers d'être britanniques. Il s'empressait de protester contre tout affront fait à l'honneur du Canada français. Quant sir Charles Tupper affirma que les Canadiens français n'étaient que des Anglais qui parlaient par hasard le français, Bourassa lui répondit que c'était précisément parce qu'ils parlaient le français qu'ils n'était pas anglais. ${ }^{12}$ Pour Henri Bourassa, le sort du Canada français se situait au cœur de son univers.

On ne peut douter de son attachement pour l'Église. Il déclarait un jour: "Ả l'Église catholique et romaine nous avons voué un amour sans bornes, une fidélité inviolable, une obéissance entière." ${ }^{13}$ L'Église représentait pour Bourassa un objet de vénération. C'était ce catholicisme fervent qui le poussait à se sentir responsable vis-à-vis de la société, convaincu que "la foi sans les œuvres est morte" ${ }^{14}$. Il s'accordait avec son collègue Olivar Asselin pour dire que l'État bien gouverné était l'une des plus belles offrandes que les hommes puissent offrir à Dieu. ${ }^{15}$

D'une fidélité très ardente à son Église, et croyant qu'en matière religieuse tous les catholiques doivent accepter les directives du clergé, Bourassa demeurait toutefois convaincu qu'en

\footnotetext{
11 Ibid.: 122.

12 Canada House of Commons Debate, June 7, 1900, 6908.

13 Henri Bourassa, Le Canada à Lourdes (Montréal, 1914), 20.

14 Henri Bourassa, Religion, langue, nationalité (Montréal, 1910), 10.

15 Olivar Asselin, Le Nationaliste, journal du 8 mai 1904, 2.
} 
matière politique l'Église accordait pleine liberté à ses enfants. ${ }^{16}$ Bourassa n'était pas castor, quoique ultramontain. Cette distinction restait significative durant la papauté de Léon XIII (18781903) qui rejetait la conception selon laquelle l'Église jouirait d'une primauté dans le domaine de la législation laïque; il attribuait plutôt à l'État une zone d'influence autonome, loin de toute influence ecclésiastique. Bourassa, en bon ultramontain, acceptait ce principe; ainsi s'en vantait-il devant Goldwyn Smith, quand il lui disait qu'il suivait la théologie de Rome mais la politique de chez lui.

De plus, rappelons que Bourassa, toujours soucieux d'attirer par sa doctrine les Canadiens de toutes croyances religieuses ${ }^{17}$, avait nettement l'intention d'édifier un mouvement laïc. $L e$ Devoir concentra son attention sur des questions politiques et sociales. Malheureusement un journal rédigé par des catholiques engagés ne pouvait qu'engendrer la méfiance chez les protestants quant aux buts ultimes de Bourassa.

L'épanouissement de la culture française en Amérique du Nord, le triomphe sur ce continent des principes catholiques, telles étaient les aspirations fondamentales qui poussaient Bourassa à agir. Pour lui, ses désirs ne devaient pas exister seulement en parallèle, mais se fondre dans une vision morale qui devenait alors le but ultime et la source la plus profonde de son énergie sociale extraordinaire. Convaincu que, comme pour d'autres peuples, les Canadiens français ont reçu une mission de Dieu, il écrit:

La Providence a voulu que le groupe principal de cette colonisation française et catholique constituât en Amérique un coin de terre à part, où l'état social, religieux et politique se rapproche le plus de ce que l'Eglise catholique, apostolique et romaine nous apprend être l'état le plus désirable des sociétés. ${ }^{18}$

La langue française demeure donc essentielle pour les Canadiens

16 Robert Rumilly, Henri Bourassa - La vie publique d'un grand Canadien (Montréal, 1953), 431.

17 Henri Bourassa, Le Devoir, 30 janvier 1911, 1.

18 Henri Bourassa, Religion, langue, nationalité (Montréal, 1910), 15. 
français engagés dans cette œuvre car "la meilleure sauvegarde de la foi, c'est la conservation de l'idiome dans lequel, pendant trois cents ans, ils ont adoré le Christ". ${ }^{19}$ Ce n'est donc pas une tâche légère que Bourassa prévoyait alors pour son peuple; il voulait que les Canadiens français deviennent les apôtres de l'Amérique du Nord, ${ }^{20}$ et que tout en édifiant un Etat idéal, ils convertissent au catholicisme un continent composé en majorité de protestants ou de libres-penseurs.

Le patriotisme canadien-français de Bourassa, de nature culturelle, était un patriotisme qui n'envisageait donc pas d'État séparé pour le Canada français. Bourassa croyait que l'épanouissement de la culture canadienne-française demeurait possible au sein de la structure politique établie lors de la Confédération.

Cette prémisse signifiait par conséquent qu'il était possible pour un Canadien français d'être fidèle à sa propre culture tout en demeurant engagé à l'égard du Dominion. C'est précisément ce dernier point que Jules-Paul Tardivel - le rédacteur de La Vérité, hebdomadaire castor - ne voulait pas reconnaître. Il affirmait que la Confédération avait eu des conséquences désastreuses pour les Canadiens français: d'une part, leurs droits se voyaient anéantis dans l'Ouest et, d'autre part, leurs hommes politiques ne pouvaient espérer jouer tout au plus qu'un rôle secondaire. Bref, la Confédération se présente comme une "machine destinée à nous écraser comme race distincte". De plus, puisque le Dominion représente une absurdité politique et géographique, sa débâcle devient inévitable. Tardivel invite les Canadiens français à se préparer au fracassement de l'union en cultivant chez leurs concitoyens un patriotisme pour le Canada français, de sorte qu'à la première occasion ils puissent rétablir "un édifice moins vaste mais plus conforme aux aspirations nationalistes de notre race". ${ }^{21}$ Par conséquent, il critique l'encouragement au nationalisme canadien dans les pages du Nationaliste:

19 Ibid., 14.

20 Ibid., 16.

21 J.-P. Tardivel, La Vérité, 15 mai 1904, 2. 
Notre nationalisme à nous est le nationalisme canadien-français ... la nation que nous voulons voir se fonder, c'est la nation canadienne-française. ${ }^{22}$

Dans sa réplique, Bourassa utilisa à dessein les mêmes formules pour indiquer nettement qu'il croyait qu'un Canadien français devait partager son patriotisme avec tous les Canadiens:

Notre nationalisme à nous est le nationalisme canadien, fondé sur la dualité des races. La nation que nous voulons voir se développer c'est la nation canadienne, composée des Canadiens français et Canadiens anglais, c'est-à-dire de deux éléments séparés par la langue et la religion mais unis dans un commun attachement à la patrie commune..$^{23}$

Il soutenait aussi que les Canadiens français devaient agir à l'intérieur des circonstances dans lesquelles l'histoire les avait placés: "notre ligne de conduite me semble toute tracée par le régime que l'Acte de la Confédération nous a fait." ${ }^{24}$

Bourassa croyait que les Canadiens français pouvaient revendiquer tout le Canada comme leur domaine culturel. Aussi longtemps que le Québec demeurait partie constituante de la Confédération, il pouvait venir en aide aux groupements français dans les autres provinces par des moyens constitutionnels ; ${ }^{25}$ par contre, la rupture de l'union précéderait leur assimilation. De plus il ne voyait aucun empêchement à ce que les Canadiens français puissent bénéficier des richesses du Dominion entier. En effet, s'ils choisissaient de demeurer isolés, ils ne perdraient que des "intérêts matériels". ${ }^{26}$ Ce que Bourassa voulait, c'est que son peuple apporte sa pierre à l'édifice national et qu'il s'y ménage la place qui lui revient de droit..27 Tardivel, lui, désirait

22 Ibid., 1er avril 1904, 5.

23 Henri Bourassa, Le Nationaliste, journal du 3 avril 1904, 2.

24 Henri Bourassa, "Le patriotisme canadien-français", Revue canadienne, XVI (no 1): 434 .

25 "Toute la pensée de Bourassa sur le séparatisme" - Un document historique. Textes inédits provenant de la correspondance personnelle de Bourassa et reproduction d'articles et conférences, L'Action nationale, LIII (mai-juin 1964) : 831 .

26 Ibid: 829.

27 Le Nationaliste, 3 avril 1904, 2. 
que les Canadiens français délaissent l'Ouest canadien et l'abandonnent à l'assimilation, qu'ils livrent Ottawa au pouvoir des Anglo-Saxons et qu'ils se limitent au Québec. Bourassa souhaitait au contraire, que les Canadiens français élargissent leurs horizons et écartent l'isolement et l'esprit de clocher. ${ }^{28}$ Il désirait que son peuple se plonge corps et âme dans le brouhaha qu'est l'édification de la nation canadienne, de sorte qu'il puisse continuer à réclamer tout le Dominion à parts égales avec les Canadiens anglais.

Bourassa demeurait confiant en l'avenir de la culture canadienne-française au sein d'un Canada dans lequel son peuple, quoique minoritaire, représentait une force politique très significative. Cependant, dans une fédération impériale anglo-saxonne de caractère mondial, l'élément canadien-français serait trop faible pour survivre. L'envoi de troupes en Afrique du Sud en 1899 ébranle Bourassa. Il est d'avis que par ce geste le Canada abdique son droit de refuser toute requête britannique d'un concours militaire. Ainsi, pensait-il, le Canada s'est engagé dans une voie qui mène à une fédération de facto.

D'ailleurs, l'âpreté au gain de l'impérialisme britannique, caractérisé par une convoitise pour la spéculation des terres et par une domination militaire, faisait croire que le secours militaire apporté au Transvaal ne serait que le premier de plusieurs sacrifices exigés par la Grande-Bretagne. Trop étendue, elle avait assumé plus de responsabilités qu'elle n'en pouvait porter et se tournait donc vers les colonies pour y solliciter des contributions militaires "en hommes et en trésors, mais surtout en hommes". ${ }^{29}$ Les associés de Chamberlain au Canada, lord Minto et le général Hutton, avec le concours du Montreal Star, avaient réussi à impliquer le Dominion dans le conflit sud-africain. Le Secrétaire pour les Colonies se vanta par la suite d'avoir pu assurer la participation de toutes les colonies dans les "guerres pour l'Empire".30 Au Canada, il se développait chez les

\footnotetext{
28 Henri Bourassa, "Le patriotisme canadien-français", Revue canadienne, XVI (no 1): 428, 430.

29 Henri Bourassa, Great Britain and Canada (Montréal, 1902), 23.

30 Ibid., 38.
} 
Anglo-Saxons un sentiment d'engagement à l'égard de l'impérialisme; la British Empire League recruta plusieurs adhérents au sein du Cabinet et parmi les députés du Parlement d'Ottawa. Le danger était grand.

Malheureusement les Canadiens anglais demeuraient insensibles devant la menace que cette poussée impérialiste représentait pour l'indépendance du Canada, et cela à cause de leur fascination pour la mère patrie. Bourassa en vint à conclure que le seul moyen de neutraliser la puissance d'attraction de l'impérialisme serait de le contrecarrer par l'idée d'un nationalisme canadien. Si l'on réussissait à convaincre les Canadiens anglais d'envisager toutes les questions impériales dans une perspective essentiellement canadienne, ${ }^{31}$ l'autonomie serait plus près de sa réalisation et le Dominion à l'abri d'une fédération impériale.

Tactique habile que de faire appel à l'esprit de nationalisme canadien chez les Canadiens anglais et façon détournée de prévenir les empiétements de l'impérialisme britannique. Avec le temps, Bourassa comprit qu'il contenait un autre bienfait majeur: le nationalisme canadien fournissait un puissant argument pour convaincre les Canadiens anglais désireux d'édifier une forte nation canadienne d'admettre le biculturalisme.

Qu'il soit question des écoles séparées de la Saskatchewan et de l'Alberta ou de la langue française en Ontario, les arguments de Bourassa demeurèrent essentiellement les mêmes. D'une part, les Canadiens français avaient droit - selon la morale et la constitution - aux écoles séparées et à l'usage du français comme langue d'enseignement; ${ }^{32}$ d'autre part, les écoles séparées et l'usage du français dans ces écoles formaient les éléments essentiels de l'édification de la nation canadienne. Ainsi, durant la crise des écoles séparées qui sévit dans les nouvelles provinces de l'Ouest, Bourassa déclare:

31 Ibid., 45.

32 Henri Bourassa, Les écoles du Nord-Ouest (Montréal, 1905), 2. P.E. Smith, Henri Bourassa and Sir Wilfrid Laurier (thèse non publiée) (Université de Toronto, 1948), 204. - Henri Bourassa, La langue française et l'avenir de notre race (Québec, 1913), 9. 
Chercher l'union des deux races, au Canada, en dehors $\mathrm{du}$ respect mutuel qu'elle doivent à leurs droits respectifs, c'est édifier la nation sur une base fragile, c'est lui donner comme pierre angulaire un élément de ruine et de destruction. ${ }^{33}$

Il reprend ce même raisonnement en 1912 quand il défend l'usage du français dans les écoles de l'Ontario. Les Canadiens français demeureront fidèles aux institutions britanniques et au Canada, si "leurs privilèges nationaux, et particulièrement leur langue, sont respectés"; par contre, le jour où les Canadiens français abandonneront tout espoir de voir respecter leurs droits, ils deviendront américains car ils ne verront plus aucun avantage à demeurer britanniques. ${ }^{34}$

En 1912 Bourassa écrit que lui et ses amis espèrent inculquer chez tous les Canadiens "un sentiment dominant de patriotisme canadien qui assurerait et orienterait les politiques du Canada dans la conduite de ses affaires internes ou de ses relations avec d'autres communautés britanniques" ${ }^{35}$. Le nationalisme canadien protégerait le Canada français contre l'impérialisme britannique et le protestantisme anglo-saxon. Bourassa et ses partisans s'identifient parfaitement quand ils se déclarent des nationalistes.

En 1903, Bourassa et ses collègues - que je nommerai ciaprès les nationalistes - rédigent un programme pour le développement $d u$ nationalisme canadien. ${ }^{36}$ On y indiquait que le nationalisme canadien pourrait être raffermi par une autonomie plus étendue du Dominion vis-à-vis de la Grande-Bretagne, par une augmentation de l'autonomie des provinces vis-à-vis du gouvernement central, et par une politique exclusivement canadienne de développement économique et intellectuel. Je me propose de traiter seulement des deux premières propositions.

33 Henri Bourassa, Les écoles du Nord-Ouest, ibid., 28.

34 Henri Bourassa, La langue française et l'avenir de notre race (Québec, 1913), 14, 18.

35 Bourassa to Belloc, August 7, 1912, Bourassa Papers.

36 Ligue nationaliste canadienne, Programme (Montréal, 1903). 
Pour ce qui est du premier thème - celui des relations impériales - les nationalistes déclarèrent que la Province avait permis que la Grande-Bretagne fasse la conquête du Canada afin que les Canadiens puissent s'habituer à jouir de la liberté qu'accordent les institutions parlementaires. En effet, le Dominion a déjà prouvé qu'il mûrissait ses aptitudes pour l'autonomie. Le Canada, pour sa part, avait déjà suffisamment courbé l'échine en fournissant un accès à ses ports et l'usage de son réseau de communications pour fins militaires. Afin de se prémunir contre d'autres empiétements britanniques, le Canada devait jouir de la plus grande mesure d'autonomie politique, commerciale et militaire compatible avec le maintien du lien colonial.

On y insistait aussi pour que le Canada continue à jouir de tous les droits politiques déjà acquis et que l'on rejette toute participation du Dominion, soit au parlement britannique, soit au conseil impérial. On devait également rendre publique toute correspondance entre le Ministère des Colonies et le Dominion afin de prévenir toute entente secrète entre Ottawa et Londres. ${ }^{37}$ Il fallait qu'on informe le Parlement de toutes conférences impériales et, si le gouvernement recevait l'approbation pour y participer, que l'on s'engage alors à assurer la publicité la plus complète quant aux délibérations et aux décisions de la conférence. Les nationalistes espéraient voir la puissance impériale remettre certains pouvoirs au Dominion. Par exemple, le Canada devait avoir $1^{\circ}$ pleine liberté pour organiser sa politique d'immigration; $2^{\circ}$ le droit d'être représenté aux congrès internationaux; $3^{\circ}$ une procédure qui permette à ses tribunaux de demeurer les derniers arbitres en cas d'appel à une loi fédérale.

Les nationalistes demandaient de plus l'autonomie commerciale, parce qu'ils croyaient que l'une des plus importantes prérogatives d'une nation, c'est d'abord de contrôler le commerce. ${ }^{38}$ Ils réclamaient pour le Canada le droit de signer ses propres traités commerciaux et de nommer les agents de son choix pour gérer ses affaires auprès d'autres pays.

37 Canada House of Commons Debates, February 17, 1902, 72.

38 Ibid. 
Le Canada, insistaient les nationalistes dans leur programme, ne devait jamais participer à aucune guerre impériale à l'extérieur du territoire canadien. Les Britanniques s'abstiendraient de tout recrutement au Canada et aucun officier de la milice canadienne n'obtiendrait un congé pour aller participer à une guerre impériale. La seule raison d'être des écoles militaires canadiennes et de la milice serait de voir à la défense du territoire canadien. Enfin, la milice canadienne serait sous le commandement d'un officier canadien.

La publication de ce programme n'était que la première étape d'une croisade en faveur de l'autonomie canadienne que les nationalistes engagèrent sur plusieurs fronts. Ils s'opposèrent à la propagande impérialiste émanant du Congrès des Chambres de Commerce de l'Empire. De son côté, Bourassa dénonça Tarte publiquement pour avoir appuyé l'idée d'un tarif impérial.

Selon toute évidence, la section qui portait sur l'autonomie ne fit aucune impression sur le public. Elle ne contenait rien qui soit particulièrement original ou radical. D'ailleurs, l'opposition publique de Laurier aux politiques de Chamberlain éclipsa la campagne des nationalistes. À l'été de 1902, le premier ministre dénonce les impérialistes qui veulent plonger le Canada dans le tourbillon du militarisme européen et, avec le plein concours des libéraux anglo-canadiens, il tint ferme contre les tentatives de Chamberlain qui voulait rattacher le Canada à Londres par des liens plus étroits. En 1904, il renvoie lord Dundonald, le commandant britannique de la milice canadienne, pour avoir critiqué en public la politique du gouvernement et nomme un Canadien pour lui succéder. Jusqu'à la retraite de Chamberlain en 1905 et du relâchement de la pression impérialiste qui s'ensuivit, c'est Laurier, plus que la Ligue nationaliste, qui articule l'opposition du Canada français à l'impérialisme.

Les nationalistes se distinguèrent à l'occasion du projet de loi navale de 1910. Laurier lui-même était l'auteur de cette politique que plusieurs personnes au Québec pensaient devoir mener le Canada vers la participation à des guerres impérialistes 
britanniques. Et ce furent les nationalistes qui dirigèrent la résistance. Cette fois, aucun doute sur l'impression qu'ils exercèrent sur le Canada français. Avec le concours des conservateurs du Québec, ils collaborèrent à la défaite de Laurier en 1911 en élisant vingt-sept candidats dits anti-Laurier.

Le nouveau premier ministre, sir Robert Borden, introduisit son propre projet de loi navale en décembre 1912, projet auquel Le Devoir s'empressa de s'opposer. Quoique la majorité libérale ait refusé le bill au Sénat en mai 1913, le Montreal Star rejette le blâme de cette défaite sur l'influence exercée par les nationalistes. Bourassa acquiesce et prétend que les nationalistes, par leur opposition à diverses propositions qui établissaient une marine canadienne, qui fournissaient des dreadnoughts à la flotte britannique ou une contribution en argent à la mère patrie, ont remporté une "victoire morale sans précédent" ${ }^{39}$ en faveur du mouvement nationaliste.

En dépit des prétentions de Bourassa, les nationalistes n'avaient eu que très peu à faire dans la défaite du projet de Borden; la majorité des nationalistes élus avaient appuyé le plan. En fin de compte, c'est l'impuissance des deux partis qui ne pouvaient s'entendre sur un compromis acceptable plutôt que l'influence des nationalistes qui avait empêché toute entreprise navale canadienne.

En 1914, le Dominion a déjà considérablement étendu son autonomie. Seulement deux objectifs importants des nationalistes demeuraient inachevés: le Conseil Privé à Londres était toujours la dernière cour d'appel pour les lois fédérales, et le Canada n'avait pas encore acquis le pouvoir absolu qui lui permettrait de conclure ses propres traités commerciaux. Que Laurier, le chef de l'un des deux grands partis, ait affirmé le droit moral du Canada de s'abstenir des guerres britanniques, c'était un indice de l'efficacité de ce mouvement. Mais le développement du nationalisme canadien était beaucoup plus redevable au succès éclatant d'une économie nationale unitaire qu'à la

${ }^{39}$ Mason Wade, The French Canadians 1760-1945 (Toronto, 1955), 633. 
faible proportion d'opinion canadienne-française que représentait le mouvement nationaliste.

Il était certain que plus d'autonomie ferait avancer le nationalisme canadien. Mais il était loin d'être évident que les nationalistes avaient raison quand ils proclamaient que le succès de la Confédération dépendait du souci avec lequel le pouvoir fédéral respectait les droits accordés aux provinces et aux minorités. Car quelle que fut la situation en 1867, vers 1900 , les gouvernements provinciaux anglo-protestants étaient devenus les citadelles du canadianisme anti-français; et plus les provinces étendaient leurs pouvoirs, moins le gouvernement fédéral devenait capable d'intervenir pour protéger les minorités canadiennes-françaises.

La section qui portait sur les relations fédérales-provinciales était destinée à satisfaire et les réclamations traditionnelles du Québec - à savoir, le plus d'autonomie politique et financière possible - et la nouvelle réclamation quant aux droits des catholiques qui revendiquaient des écoles séparées dans les nouvelles provinces de l'Ouest, réclamation dont on ne pouvait guère présumer, comme l'avait démontré l'incident regrettable du Manitoba. Dès 1914, les provinces avaient considérablement accru leurs pouvoirs. Ottawa avait cessé ses interventions et accordé de fortes concessions financières. Mais l'accroissement d'autonomie provinciale ne fit rien pour prévenir l'assaut contre les écoles séparées dans l'Ouest et la proscription presque totale $\mathrm{du}$ français dans les écoles de l'Ontario. Plusieurs Canadiens anglais secondèrent ces mesures au nom de l'unité nationale.

L'insuccès le plus important des nationalistes se trouve ici. Ces derniers soutenaient que les Canadiens anglais ne pourraient gagner les Français à la cause du patriotisme canadien qu'en acceptant le droit de la culture française de coexister avec la culture anglaise à travers tout le Canada; mais ils ne réussirent jamais à convaincre la majorité anglaise qu'il existait une relation aussi étroite entre le biculturalisme et l'édification d'une nation canadienne. À toutes fins pratiques, les Canadiens français demeurèrent essentiellement limités à leur "réserve" du Québec. 
Pour résumer, l'objectif de Bourassa était de fonder une nation canadienne, mais la force motrice qui l'acheminait vers ce but provenait plus d'un amour pour le Canada français et d'un attachement pour l'Église catholique que d'un patriotisme pour le Dominion. Son engagement principal s'appuyait sur ce qu'il croyait être la mission du Canada français. Il lui semblait, durant la première décennie du vingtième siècle, que le succès de cette mission était menacé par la pression grandissante provenant de l'impérialisme britannique et par la poussée du Canada anglais contre un biculturalisme appliqué au Dominion entier. Comme il voulait mettre le Canada français à l'abri de l'impérialisme britannique et $\mathrm{du}$ protestantisme anglo-canadien, il préconisa un nationalisme canadien qui serait autonome à l'égard de l'empire britannique mais biculturel chez lui.

Préconiser un tel nationalisme à l'échelle nationale s'accordait parfaitement avec un patriotisme canadien-français intense parce que ce dernier était de nature culturelle et non politique; c'est donc dire que Bourassa n'a jamais proposé un Etat séparé pour le Canada français. Ainsi, il pouvait être à la fois un nationaliste politique - pour le Dominion - et un nationaliste culturel — vis-à-vis du Canada français. 